\title{
AVALIAÇÃO DA UTILIZAÇÃO DE DIFERENTES AGLOMERANTES NAS PROPRIEDADES MECÂNICAS DE PELOTAS AUTORREDUTORAS DE PÓ DE ACIARIA*
}

\author{
Anderson Azevedo Agra ${ }^{1}$ \\ Helioir Antonio da Silva Júnior ${ }^{1}$ \\ Matheus Frederico Rückert ${ }^{1}$ \\ Ligia dos Santos de Oliveira ${ }^{1}$ \\ Felipe Buboltz Ferreira ${ }^{2}$ \\ Bruno Deves Flores ${ }^{3}$ \\ Antônio Cezar Faria Vilela
}

\section{Resumo}

A geração de resíduos siderúrgicos é um problema que abrange dois cenários: o ambiental, no que diz respeito ao descarte de resíduos no meio ambiente e também tange o econômico, pois trata-se de material não reaproveitado que deve ser destinado a aterros industriais, gerando custos. Tendo isto em vista, a reciclagem desses possíveis coprodutos para a reutilização como matéria-prima do processo torna-se uma alternativa pertinente. Uma das formas de recuperação do pó de aciaria elétrica (PAE), resíduo abordado neste trabalho, é através da pelotização. Para o reaproveitamento de metais de interesse do pó foi utilizado um agente redutor, o coque de petróleo. No entanto, para formação das pelotas um aglomerante se faz necessário. Este, em associação com as partículas do óxido, tem como objetivo conferir resistência mecânica adequada às solicitações que o processo de fabricação do aço exige. Neste trabalho, são feitas considerações acerca do uso de seis composições diferentes de aglomerantes com o objetivo de avaliar o desempenho destes, através de ensaios de resistência a compressão, testes de queda e tamboramento. Como resultado, pode-se verificar o melhor desempenho da combinação da cal com cinza de casca de arroz.

Palavras-chave: Resistência mecânica; Pelotização; PAE; Aglomerantes.

\section{EVALUATION OF THE UTILIZATION OF BINDERS IN THE MECHANICAL PROPERTIES OF SELF-REDUCING PELLETS FROM EAF DUST}

\begin{abstract}
The production of siderurgical residues is a problem that covers two main scenarios: the environmental, regarding the residues' disposal on soil and also the economic field, because it's a non reused material that must be send to industrials landfills, generating costs. Thus, recycling these possible co-products to use as raw material of the process becomes a relevant option. One of the ways to recover the EAF dust, residue approached in this paper, is through pelletization. For the reutilization of the metals of interest in the dust a reducer agent was used, the petroleum coke. Nevertheless, for the pellets' formation, a binder is needed and in association with the oxides particles aims to give mechanical strength suitable for the iron making process. In this paper six different binders' composition are tested, aiming evaluate their performance through compressive strength testing, drop shatter test and tumbler test. As result it was possible to verify the efficiency of the combination hydrated lime with rice rusk ash for the three tests.
\end{abstract}

Keywords: Mechanical strenght; Pelletization; EAF dust; Binders.

Graduando de Eng. Metalúrgica, LaSid, DAMET, EE, UFRGS, Porto Alegre, RS, Brasil.

Eng. Materiais, Mestrando, LaSid, PPGE3M, EE, UFRGS, Porto Alegre, RS, Brasil.

Eng. Metalúrgico, Doutorando, LaSid, PPGE3M, EE, UFRGS, Porto Alegre, RS, Brasil.

Prof. Dr-Ing., LaSid, DAMET, PPGE3M, EE, UFRGS, Porto Alegre, RS, Brasil. 


\section{INTRODUÇÃO}

Durante a fabricação de aço em usinas siderúrgicas semi-integradas são gerados diversos tipos de resíduos sólidos, dentre eles, destacam-se as carepas, escórias e o pó de aciaria elétrica (PAE). Este último resíduo é gerado durante a fusão e refino dos aços nos fornos elétricos a arco (FEA), onde se estima que entre 1 e $2 \%$ da carga carregada no FEA torna-se PAE. Atualmente cerca de $50 \%$ do PAE gerado no mundo acaba sendo destinado a aterros industriais [1], representando um passivo ambiental, além de um grande custo de destinação para as empresas. No Brasil o resíduo é considerado perigoso segundo a NBR 10004, tornando o problema ainda mais grave em termos de destinação [2].

A composição química do PAE pode variar consideravelmente dependendo do tipo de aço fabricado em cada usina [3]. No entanto, estudos apontam que geralmente este resíduo é composto basicamente por óxidos metálicos, com presença majoritária de elementos como zinco e ferro [3;4], além de menores teores de chumbo, cádmio, estanho, etc. A presença de tais elementos em concentrações altas ou moderadas, torna o resíduo atrativo do ponto de vista da reciclagem.

Chang [5] relata a existência de um grande número de tecnologias desenvolvidas e aplicadas para a reciclagem do PAE, subdividindo-as em três grupos: pirometalúrgicas, hidrometalúrgicas e de vitrificação. Atualmente, os processos pirometalúrgicos são os mais empregados no mundo. De forma geral, nesses processos a recuperação dos metais de interesse se dá através da redução carbotérmica dos óxidos metálicos. $O$ processo Waelz é, hoje, o principal processo para a reciclagem de PAE [1]. Neste processo pelotas autorredutoras contendo PAE, são carregadas em um forno rotativo visando principalmente a geração de concentrados de zinco.

Apesar da existência de soluções tecnológicas para a reciclagem de PAE em usinas siderúrgicas semi-integradas, a reciclagem se torna um desafio quando estas não possuem unidades de redução, ou que não tem a possibilidade de destinar o seu resíduo para plantas específicas de recuperação de PAE. Buzin [6] e Bagatini [7] estudaram a possibilidade de se recuperar resíduos ferrosos através do uso de briquetes autorredutores diretamente em fornos elétricos à arco. Esses autores sugerem que tal abordagem surge como uma alternativa para caso onde não haja unidades de redução. Assim a introdução de aglomerados autorredutores pode ser uma possibilidade interessante para a recuperação de PAE $[6,7]$.

A autorredução consiste basicamente na mistura íntima de óxidos metálicos e materiais carbonosos de modo que, quando sob aquecimento em elevadas temperaturas, o teor de carbono no aglomerado seja suficiente para provocar a redução dos óxidos [7]. A integridade física dos aglomerados antes e durante o aquecimento é fundamental para o bom desempenho do processo.

Neste sentido, os aglomerantes desempenham um papel muito importante no processo de aglomeração, pois, em associação com as partículas ultrafinas dos óxidos, servem para melhorar as propriedades das pelotas úmidas, secas ou queimadas [8, 9]. $\mathrm{Na}$ pelotização, eles atuam aumentando a fase líquida dentro dos capilares, mantendo a coesão das pelotas verdes e fornecendo resistência mecânica às pelotas secas [8,9]. Existem diferentes tipos de aglomerantes e, eles podem ser divididos em: orgânicos e inorgânicos, além da combinação entre eles. Aglomerantes orgânicos, em contraste com os inorgânicos, têm a vantagem de serem eliminados durante o aquecimento e consequentemente, não contaminam o produto [9]. Em contrapartida, esses aglomerantes produzem pelotas com menor resistência a compressão, além de ter um custo elevado [4]. 
O objetivo do presente trabalho é avaliar o desempenho de diferentes aglomerantes nas propriedades mecânicas de pelotas autorredutoras de pó de aciaria elétrica. Esse estudo busca dar suporte a reciclagem do PAE através do uso de aglomerados autorredutores em forno elétrico a arco.

\section{MATERIAIS E MÉTODOS}

Nos próximos itens serão apresentadas características dos materiais utilizados, formas de fabricação e ensaios realizados.

\subsection{Matérias-Primas}

\subsubsection{Pó de aciaria elétrica}

A Tabela 1 apresenta a composição elementar do PAE, obtida a partir da caracterização via Espectrometria por Plasma Acoplado Indutivamente (ICP), onde nota-se que zinco e ferro são componentes majoritários da amostra. A Figura 1 apresenta a análise de difração por raios $X$, a qual demostra que as espécies cristalinas franklinita, zincita e magnetita são majoritárias no PAE. A Figura 2 apresenta a distribuição granulométrica do $P A E$, realizada por difração de laser.

Tabela 1. Composição química do PAE.

\begin{tabular}{ccccccccc}
\hline & $\mathrm{Zn}$ & $\mathrm{Fe}$ & $\mathrm{Mn}$ & $\mathrm{Ca}$ & $\mathrm{Mg}$ & $\mathrm{Pb}$ & $\mathrm{Na}$ & $\mathrm{C}$ \\
Composição & 34,23 & 22,80 & 2,75 & 2,49 & 1,76 & 1,10 & 1,00 & 0,91 \\
\cline { 2 - 9 } elementar & $\mathrm{K}$ & $\mathrm{Al}$ & $\mathrm{Cu}$ & $\mathrm{Ba}$ & $\mathrm{Cd}$ & $\mathrm{Ni}$ & $\mathrm{Sn}$ & \\
(\% em massa) & 0,97 & 0,20 & 0,15 & 0,05 & 0,03 & 0,01 & 0,01 & \\
\hline
\end{tabular}

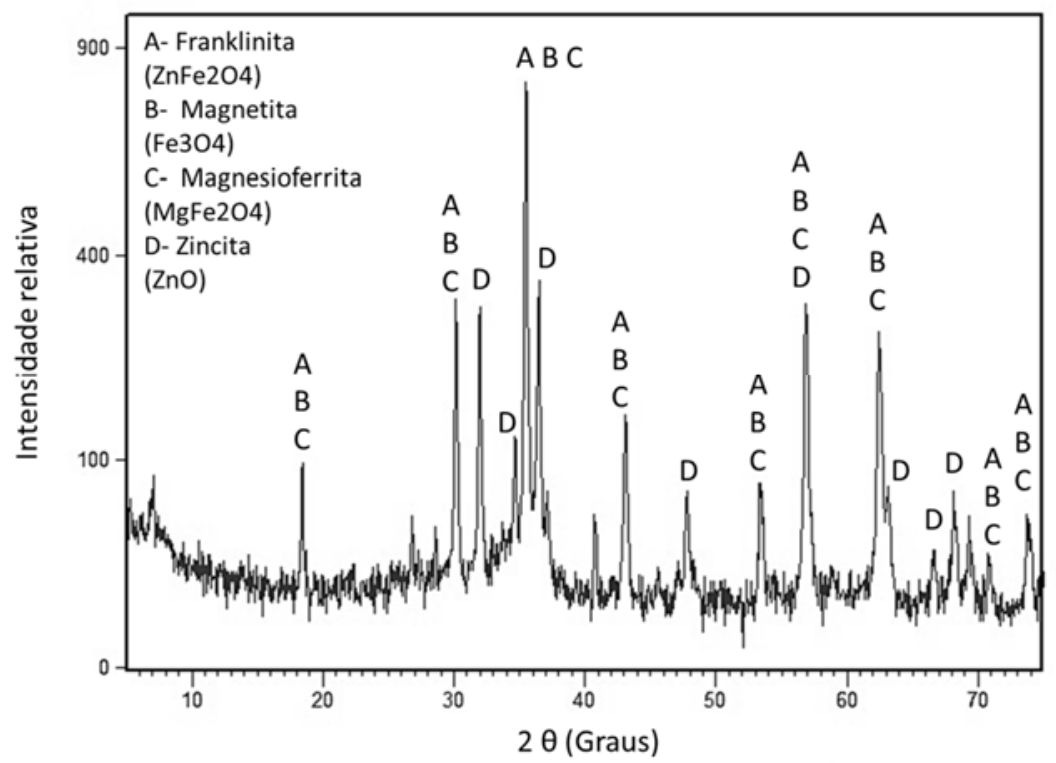

Figura 1. Difração de Raios $X$ do PAE. 


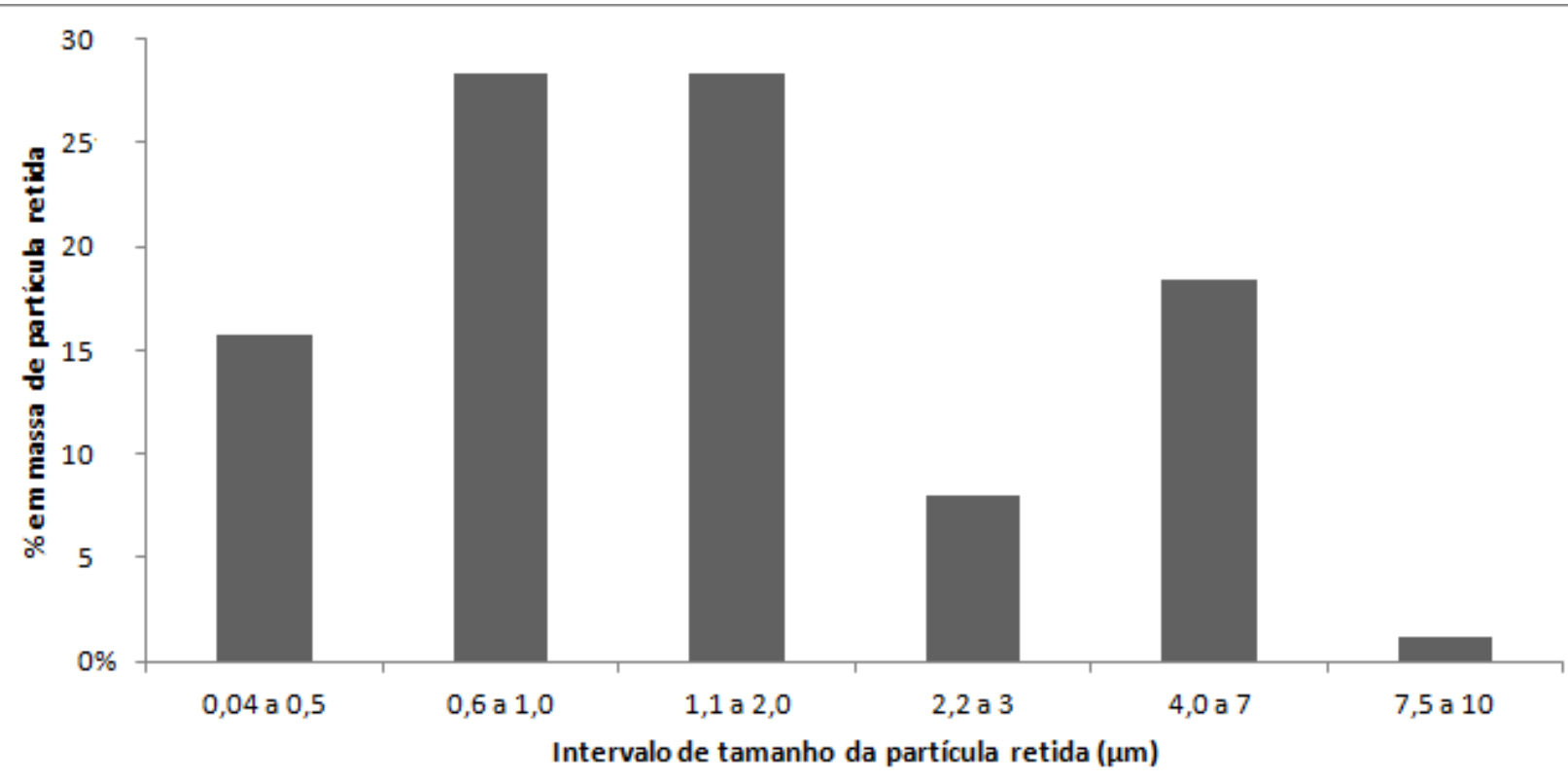

Figura 2. Distribuição granulométrica do PAE.

\subsubsection{Coque de petróleo}

A Tabela 2 mostra o resultado da análise imediata do coque de petróleo. Já a Figura 3 apresenta a distribuição granulométrica do PET, realizada por difração de laser.

Tabela 2. Análise Imediata do Coque de Petróleo, porcentagem em massa (NBR8290).

\begin{tabular}{|c|c|c|}
\hline \multicolumn{3}{|c|}{ Base seca (\% massa) } \\
\hline Matéria Volátil & Cinzas & Carbono Fixo \\
\hline 10,06 & 0,63 & 89,31 \\
\hline
\end{tabular}

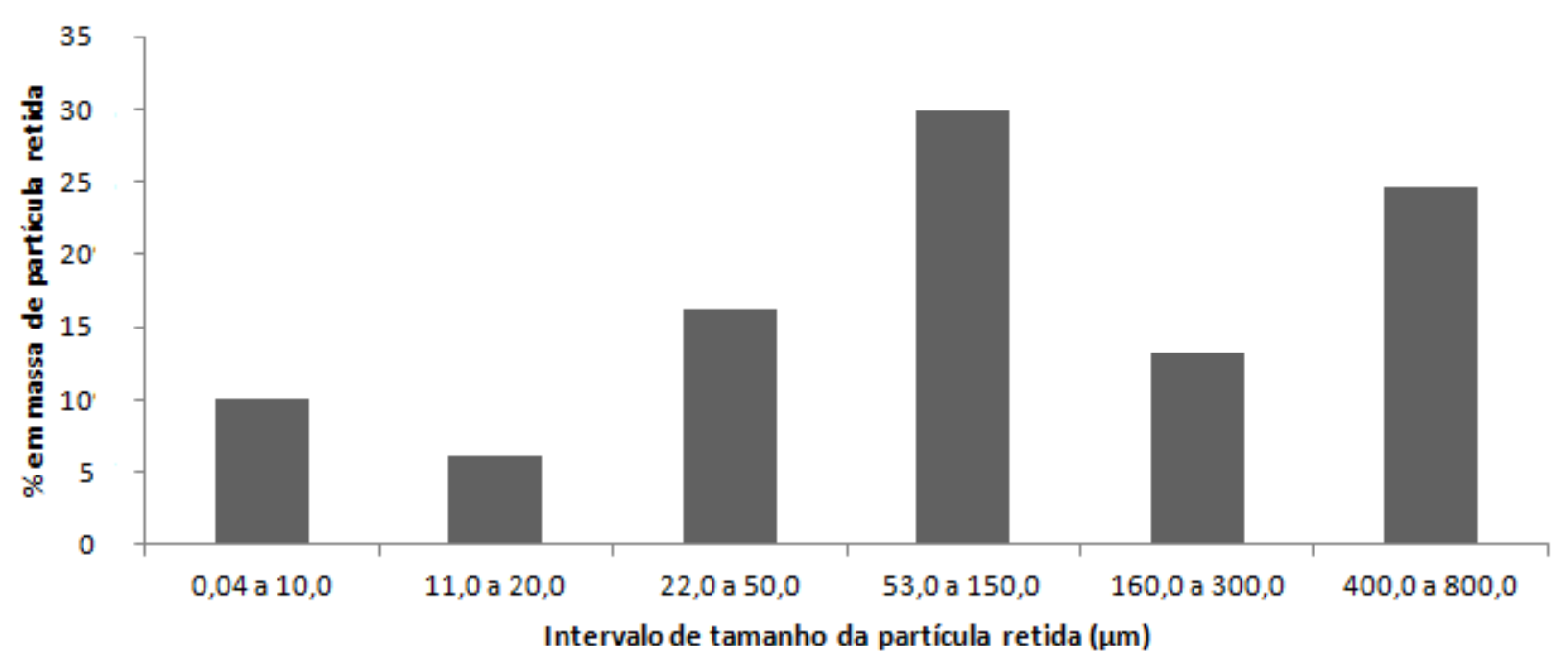

Figura 3. Distribuição granulométrica do Coque de Petróleo.

\subsection{Aglomerantes}

Foram utilizados seis diferentes composições de aglomerantes. Sendo quatro aglomerantes inorgânicos, um orgânico e uma combinação de ligantes inorgânicos. Os ligantes inorgânicos usados foram: Cal Virgem, Cal Hidratada, Cinza de casca de arroz e Cimento Portland, sendo este último utilizado em duas concentrações diferentes. $\mathrm{O}$ 
ligante orgânico utilizado foi a Dextrose. E finalmente, a combinação de cal hidratada com cinza de casca de arroz.

\subsection{Produção das Pelotas Autorredutoras}

Para a preparação da pelotização foi feita uma mistura inicial (PAE + PET) de relação 9:1 (em massa) de PAE. Após, foi acrescentado à mistura os aglomerantes (conforme Tabela 3). Posteriormente a preparação das matérias-primas, adicionou-se água em aproximadamente $20 \%$ em massa e as pelotas foram modeladas manualmente em um diâmetro aproximado de $10 \mathrm{~mm}$.

Tabela 3. Porcentagem mássica dos aglomerantes na mistura das pelotas.

\begin{tabular}{|c|c|c|c|c|}
\hline \multirow{2}{*}{$\begin{array}{c}\text { Nome da } \\
\text { amostra }\end{array}$} & Aglomerante & \multicolumn{3}{c|}{ (\% em massa) } \\
\cline { 2 - 5 }$(5 \mathrm{Cl})$ & Cimento & 5 & 85,5 & 9,5 \\
\hline$(\mathrm{CAH})$ & Cal Hidratada & 10 & 81,0 & 9,0 \\
\hline$(\mathrm{DEX})$ & Dextrose (DEX) & 3 & 87,3 & 9,7 \\
\hline$(10 \mathrm{Cl})$ & Cimento & 10 & 81,0 & 9,0 \\
\hline$(\mathrm{CZC})$ & Casca de Arroz + Cal Hidratada & $7+3$ & 81,0 & 9,0 \\
\hline$(\mathrm{CaO})$ & Cal Virgem & 10 & 81,0 & 9,0 \\
\hline
\end{tabular}

\subsection{Ensaios de Caracterização Mecânica das Pelotas}

\subsubsection{Ensaio de compressão}

O teste de compressão tem o objetivo de avaliar o desempenho mecânico das pelotas frente a possíveis esforços durante armazenamento, bem como, preparação da carga do cestão. Para isso, pelotas com 7, 14 e 21 dias de cura foram submetidas a ensaios de compressão em uma máquina de ensaios universal Shimadzu Autograph AG-X 50 kN Shimadzu Autograph AG-X 50KN, localizada no Laboratório de Materiais Cerâmicos (LACER). Os testes foram conduzidos com velocidade de avanço constante de $0,1 \mathrm{~mm}$ min-1. A resistência a compressão das pelotas foi determinada a partir do valor médio de 10 repetições do ensaio de compressão para cada amostra.

\subsubsection{Teste de queda e tamboramento}

As amostras foram submetidas a testes de queda e tamboramento com o objetivo de avaliar as degradações das pelotas frente a situações comuns em uma usina siderúrgica, tais como, transporte, manuseio e carregamento do forno.

$O$ teste de queda foi realizado utilizando um equipamento que consiste em uma caixa (400 mm x $310 \mathrm{~mm} \times 210 \mathrm{~mm}$ ) com fundo móvel, a qual, quando acionada, permite a queda livre das pelotas de uma altura de 1,72 $\mathrm{m}$. As pelotas ao caírem se chocam contra uma chapa de aço com $13 \mathrm{~mm}$ de espessura, fixa no chão. O teste foi realizado com aproximadamente $120 \mathrm{~g}$ de pelotas. O procedimento realizado foi de três quedas consecutivas. Em cada queda, as pelotas foram peneiradas, utilizando peneiras de aberturas 9,52, 4,76, 2,83 e 1,18 mm, avaliando sua massa afim de verificar as degradações destas.

O teste de tamboramento foi realizado em um tambor tipo-I com comprimento de 700 $\mathrm{mm}$ e diâmetro interno de $130 \mathrm{~mm}$. Utilizou-se 34 pelotas, sendo estas submetidas a 100 revoluções, com velocidade de 20 R.P.M.. Após as consecutivas quedas as pelotas foram peneiradas, utilizando as mesmas peneiras do teste de queda, e avaliadas suas massas para mensurar as degradações. 


\section{RESULTADOS E DISCUSSÃO}

\subsection{Resultados}

\subsubsection{Teste de resistência a compressão}

Os resultados de resistência a compressão das 6 amostras de pelotas produzidas são apresentados na Figura 4.

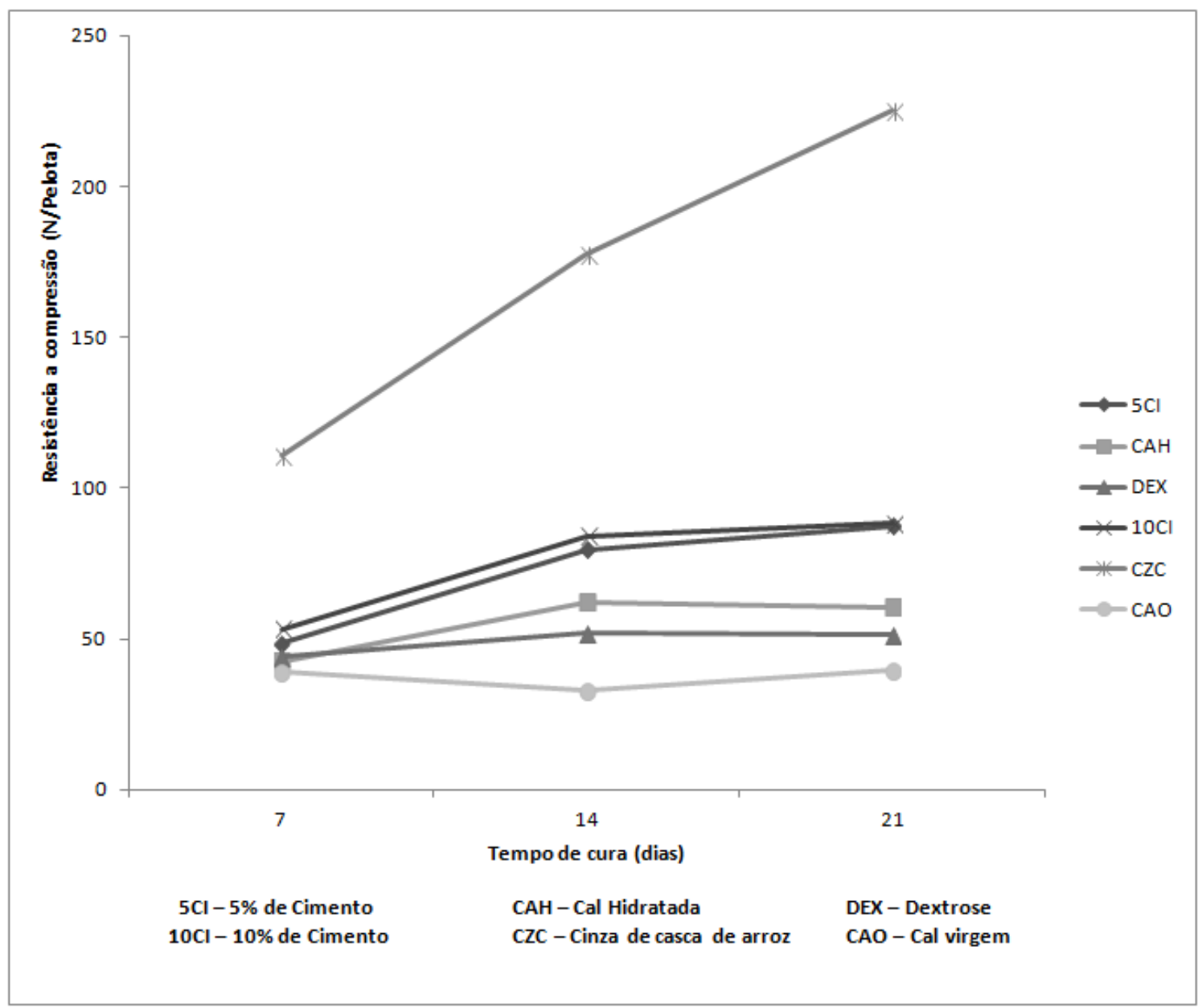

Figura 4. Resistência a compressão (N/Pelota) médio $x$ tempo de cura das pelotas (semanas).

$\mathrm{Na}$ tabela 4 são apresentados valores médios da resistência a compressão de cada composição nas três semanas de teste.

Tabela 4.Valor médio da resistência a compressão.

\begin{tabular}{|c|c|c|c|}
\hline & \multicolumn{3}{|c|}{ Resistência a compressäo média (N/pelota) } \\
\hline Aglomerante & Semana 1 & Semana 2 & Semana 3 \\
\hline $5 \mathrm{Cl}$ & 48 & 80 & 88 \\
\hline $\mathrm{CAH}$ & 43 & 62 & 60 \\
\hline $\mathrm{DEX}$ & 44 & 52 & 51 \\
\hline $10 \mathrm{Cl}$ & 53 & 84 & 88 \\
\hline $\mathrm{CZC}$ & 111 & 178 & 225 \\
\hline $\mathrm{CAO}$ & 39 & 33 & 40 \\
\hline \multicolumn{4}{|l}{} \\
\hline
\end{tabular}




\subsubsection{Teste de queda}

$\mathrm{Na}$ Figura 5 são apresentados os percentuais em massa dos fragmentos das pelotas restantes nas peneiras após sofrerem a última de três quedas consecutivas no teste de queda. Quanto maior a concentração dos fragmentos de pelotas nas peneiras de maiores aberturas, melhor o desempenho frente à este teste, visto que estes aglomerados se manteriam próximos a sua integridade necessária para as aplicações desejadas.

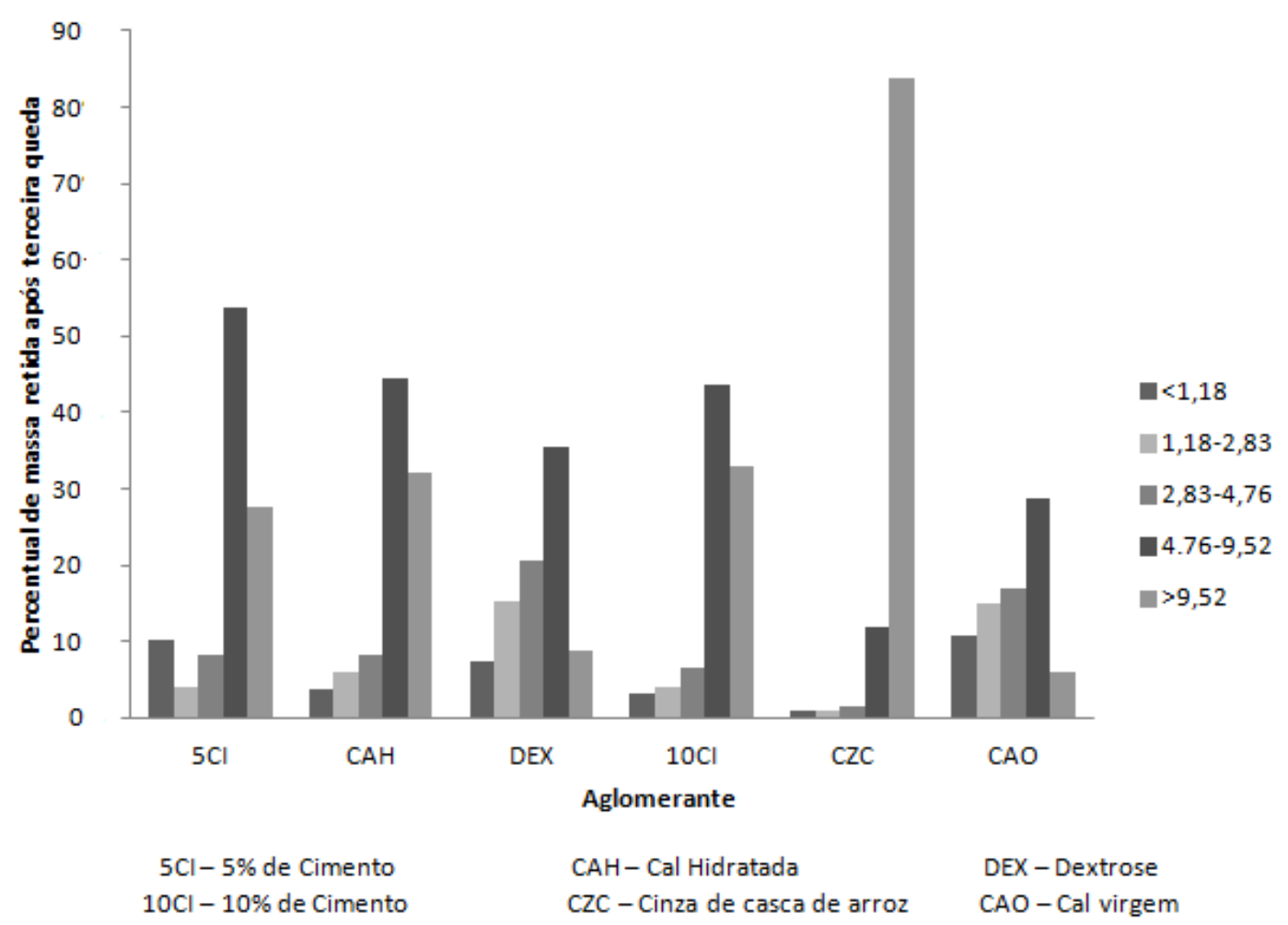

Figura 5 - Resultados do teste de queda.

\subsubsection{Teste de tamboramento}

$\mathrm{Na}$ Tabela 5 são apresentados os valores de porcentagem de massa retidos nas faixas de tamanhos descritas no teste de tamboramento.

\begin{tabular}{c|c|c|c|c|c|}
\multicolumn{7}{c}{ Tabela 5 - Resultado para o teste de tamboramento. } \\
\hline & $<1,18$ & $1,18<0<2,83$ & $2,83<0<4,76$ & $4,78<0<9,52$ & $>9,52$ \\
\hline $5 \mathrm{Cl}$ & $26,60 \%$ & $4,66 \%$ & $3,87 \%$ & $31,62 \%$ & $33,25 \%$ \\
\hline $\mathrm{CAH}$ & $48,48 \%$ & $4,33 \%$ & $4,90 \%$ & $18,88 \%$ & $23,44 \%$ \\
\hline $\mathrm{DEX}$ & $56,79 \%$ & $14,19 \%$ & $13,93 \%$ & $15,08 \%$ & $0,00 \%$ \\
\hline $10 \mathrm{Cl}$ & $43,91 \%$ & $6,33 \%$ & $6,44 \%$ & $32,27 \%$ & $11,09 \%$ \\
\hline $\mathrm{CZC}$ & $12,05 \%$ & $0,00 \%$ & $0,00 \%$ & $0,00 \%$ & $87,98 \%$ \\
\hline $\mathrm{CAO}$ & $67,53 \%$ & $10,66 \%$ & $10,26 \%$ & $11,57 \%$ & $0,00 \%$ \\
\hline
\end{tabular}

Na Figura 6 são apresentados os valores de geração de finos $(<1,8 \mathrm{~mm})$ de cada mistura, após o teste de tamboramento. 


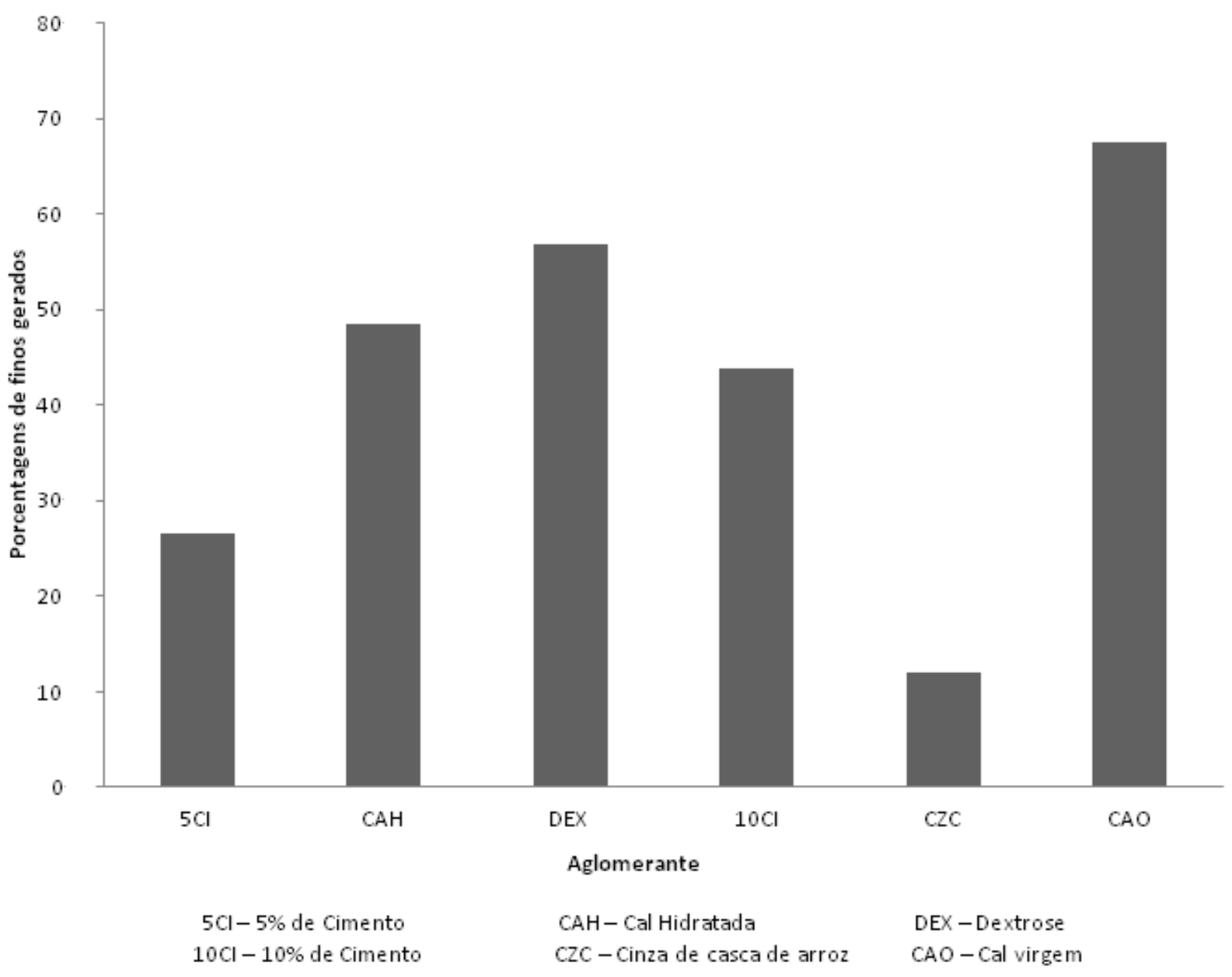

Figura 6 - Porcentagem de finos gerados no teste de tamboramento.

\subsection{Discussão}

Todos os testes realizados tiveram como objetivo avaliar as propriedades mecânicas das pelotas, portanto a interpretação dada aos resultados obtidos para todos os testes partem do mesmo princípio. Algumas considerações para estes resultados são feitas a seguir:

Pode-se observar que o aglomerante com composição 3\% de Cinza de Casca de Arroz e $7 \%$ de Cal Hidratada (CZC) possui um melhor desempenho em todos os testes realizados (Figuras 5, 6 e 7). Este desempenho deve-se, provavelmente, a reação que o material pozolânico realiza com o hidróxido de cálcio, na presença de água, formando então silicatos de cálcio estáveis com boa capacidade cimentante [11].

As pelotas que se saíram melhores no teste de tamboramento (geraram menos finos) foram as que possuem como aglomerante a mistura de cinza de casca de arroz com cal hidratada e $5 \%$ de cimento, respectivamente (Figura 7).

A resistência adquirida pelas pelotas que utilizam cimento como aglomerante se deve a dois mecanismos: a hidratação dos silicatos e a formação de carbonatos. [12]

Observa-se que pelotas que tiveram o cimento (5 e 10\%) utilizado como aglomerante obtiveram desempenhos muito semelhantes (Tabela 4). Segundo Lea [10], existe uma quantidade ótima de cimento para obtenção da melhor propriedade mecânica possível. Essa quantidade está relacionada à formação de uma camada superficial entre as partículas. Quantidades de cimento acima deste valor, ou seja, que formam mais de uma camada sob as partículas, não aumentarão a resistência final do aglomerado. 
A resistência mecânica das pelotas, que tem cal hidratada como aglomerante, deve-se a reação de recombinação que ocorre do gás carbônico do ar com o hidróxido, formando o carbonato inicial, cujos cristais formados ligam de maneira permanente os grãos do agregado utilizado.

Portanto, o endurecimento das pelotas com cal acontece de fora para dentro, exigindo certa porosidade da pelota, que proporciona a evaporação da água e a penetração do gás carbônico [13].

As pelotas que apresentaram maior geração de finos foram as que possuem Cal virgem como aglomerante, seguida pelas pelotas com Dextrose (Tabela 5).

O intuito das pelotas, neste trabalho avaliadas, é a reutilização no FEA. Porém por não existir um estudo sobre as propriedades exigidas para o processo não se sabe se a resistência obtida como boa, por exemplo, da CZC é suficiente para o processo. Pode, também, a resistência mecânica ter sido acima do necessário, podendo então reduzir o percentual de aglomerante, obtendo-se assim pelotas com igual capacidade de uso no processo e menor custo.

\section{CONCLUSÕES}

Com base nos resultados dos testes pode-se concluir que:

a) O melhor desempenho nos testes foi obtido pela mistura de cal hidratada com cinza de casca de arroz (CZC), no teste de resistência à compressão estas pelotas obtiveram força de ruptura média de 225 N/Pelota, na terceira semana de cura;

b) Os resultados semelhantes das duas composições de cimento, em testes como o de resistência à compressão, demonstram a vantagem econômica na utilização da mistura com menor percentual de cimento;

c) Cal virgem e Dextrose como aglomerantes não obtiveram bons resultados.

\section{Agradecimentos}

Os autores agradecem ao professor Antônio C. F. Vilela, Coordenador do Laboratório de Siderurgia, pela oportunidade de experiência junto ao laboratório, à Fundação Luiz Englert pelo auxílio financeiro à pesquisa, à Universidade Federal do Rio Grande do Sul, ao LaSid (Laboratório de Siderurgia), ao O Laboratório de Materiais Cerâmicos (LACER), pela disponibilidade do uso do equipamento para a realização do teste de compressão. Aos bolsistas de iniciação cientifica Bruno G. Gottschefsky, Cássio Carvalho e Matheus C. S. Munhoz pelo apoio na execução dos testes de caracterização mecânica das pelotas.

\section{REFERÊNCIAS}

1 Juergen Ruetten; Ron Crittendon. Environmental Friendly recycling of EAF steel milldust GSD'S state-of-the-art Waelz Process

2 J. MacCrea and C.A. Pickles: Proc. Electric Furnace Conf., Vol. 53, ISS, Warrendale, PA, (1995), 159.

3 Machado, J. G. M. S., Estudo de caracterização e avaliação da influência do tempo na redução carbotérmica do pó de aciaria elétrica (Dissertação de Mestrado), Porto Alegre, 2004.

4 Adolfo P. Zambrano; Cyro Takano; Marcelo B. Mourão; Solon Y. Tagusagawa. Influence of the Binder on the Mechanical Properties of the Chromite Self-Reducing Pellets. IJBHT.2013 
5 Chang, H. S.: Taiwan 1999 Educational Programme on Waste Treatment of EAF Slag and Dust, Taichung, Taiwan, 19-21 de outubro de 1999, Taiwan Steel \& Iron Industries Association, Taipei, Taiwan, 1999.

6 Buzin, P. J. W. K., Desenvolvimento de briquetes autorredutores a partir de carepas de processamento siderúrgico para utilização em forno elétrico a arco (Dissertação de mestrado), Porto Alegre, 2009.

7 Bagatini, M. C., Estudo da reciclagem de carepa através de briquetes autorredutores para uso em forno elétrico a arco (Tese de doutorado), Porto Alegre, 2001.

8 Kurt Meyer. Pelletizing of Iron Ores. 1980.

9 Sujay Kumar Dutta, RameshwarSah, YakshilChokshi. Iron Ore - Coal / Coke Composite Pellets. LAMBERT. 2013.

10 Lea, F. M., The chemistry of cement and Concrete, Chemical Publishing Company, New York, 1971.

11 A. M. Neville, Properties of concrete, Pitman Publishing, London (1975).

12 Alberto E. A. Nogueira., Estudo da redução carbotérmica de minérios de ferro na forma de pelotas ou misturas autorredutoras em forno rotativo. São Paulo, 2010.

13 Oliveira. H.M. Aglomerantes. In: BAUER, L.F.A (Org). Materiais de Construção I. 5 ed. Rio de Janeiro: LTC, 2008. 